\title{
Michel Cartry : l'homme et le maître à penser
}

Tal Tamari

\section{(2) OpenEdition}

\section{Journals}

Édition électronique

URL : http://journals.openedition.org/span/1647

DOI : $10.4000 /$ span. 1647

ISSN : 2268-1558

\section{Éditeur}

École pratique des hautes études. Sciences humaines

\section{Édition imprimée}

Date de publication : 1 janvier 2014

Pagination : 147-150

ISBN : 9782909036434

ISSN : 0294-7080

\section{Référence électronique}

Tal Tamari, « Michel Cartry : l'homme et le maître à penser », Systèmes de pensée en Afrique noire [En ligne], 19 | 2014, mis en ligne le 04 février 2020, consulté le 10 février 2020. URL : http:// journals.openedition.org/span/1647 ; DOI : 10.4000/span.1647 


\section{Michel Cartry : \\ l'homme et le maître à penser*}

Tal Tamari

Directrice de recherche au CNRS Institut des mondes africains (IMAF)

Je voudrais témoigner de la qualité de l'enseignement et du rayonnement de Michel Cartry, que j'ai eu le privilège de connaître à partir de 1978, d'abord dans le cadre de son séminaire, consacré aux religions de l'Afrique, puis en tant que directeur de ma thèse.

Permettez-moi d'évoquer quelques souvenirs personnels, datant de l'époque où j'ai fait sa connaissance.

Alors étudiante en Angleterre, à Oxford, j'avais écrit à Germaine Dieterlen en lui faisant part des réflexions que m'inspiraient ses travaux sur les mythes et signes bambara et dogon. Avec une extrême gentillesse, elle me répondit en m'invitant à la rencontrer à Paris. Là, elle m'expliqua qu'elle ne dirigeait plus de travaux, mais me recommanda d'assister au séminaire de Michel Cartry, qui lui avait succédé dans l'enseignement à l'EPHE. Comme j'ai pu le constater par la suite, elle devait participer activement à ce séminaire pendant plusieurs années encore.

Quant à moi, j'allais assister à ce séminaire jusqu'au début des années 1990, bien après la fin de mon cursus formel, car Michel Cartry ne se répétait pas.

Tout le monde se souvient de l'atmosphère intellectuelle électrique qui régnait à Paris à la fin des années 1970. Des personnes de toutes disciplines et de tous horizons affluaient, souvent sans inscription aucune, dans les cours et séminaires universitaires.
* Transcription de l'allocu. tion lue lors du colloque, le 15 octobre 2011. 
${ }^{1}$ Dans ses séminaires, M. Cartry s'attacha surtout aux travaux de J. Goody portant sur l'initiation Bagré et les rites funéraıres; déjà à l'époque pourtant. J. Goody étaıt reconnu principalement pour ses travaux sur l'impact social de l'écriture.

${ }^{2}$ On ne pourra mentionner ic que ses ouvrages Les Bobo: nalure et fonction des masques. Paris: ORSTOM, 1980, $2^{\mathrm{e}}$ éd. légèrement revue: Tervuren: Musée royal de l'Afrique centrale, 1999 et Masques bobo: vie, formes et couleurs, Tervuren: MRAC. 2008. aınsi que le film Hivernage à Kouroumani, CNRS Audıvi. suel. 1978. On lui associera le souvenır d'O. Gollnhofer (1934.94), spécıalıste du Gabon, particulièrement noté pour ses études sur les rites initiatiques Bwiti. Depuis que cette allocution a été prononcée. on a eu à déplorer la perte de: R. Sillans (nov. 2011), ethnobotaniste et historien du Gabon - ces deux chercheurs spécialistes d'un même pays ont souvent travaillé en collaboration L. de Heusch (août 2012), cofondateur et premier directeur du laboratoire, qui gràce à ses fines analyses comparatıves des mythes du continent afrıcain et ceux de l'aire culturelle bantoue en partıculier, justíía et réintroduit (à la suite de J. Frazer) le concept de «royauté sacrée» en anthropologie; J.F. Vincent (déc. 2012), spécialiste du Tchad, du Cameroun, de la royauté sacrée et du statut des femmes: Y. T. Cissé (déc. 2013), connaisseur inégalé des sociétés mandingues, pionnier de la collecte et de l'interprétation des traditions orales, et fin humaniste. On leur associera encore le souvenir de M. Izard (tév. 2012), proche amı de $M$ Cartry et du laboratorre, historien des royaumes moss (Burkina Faso) et théoricien du politique.
Le séminaire de Michel Cartry se distinguait par le souci de la mise en relation de ses données de terrain, recueillies auprès des Gourmantché du Burkina Faso, avec celles, rapportées dans les écrits d'autres chercheurs, qu'il estimait pertinentes. Et notamment celles de Jack Goody, alors professeur à Cambridge, qui étudiait certaines populations, également de langue voltaïque, du Ghana voisin ${ }^{1}$.

Je n'ai jamais auparavant, ni depuis, assisté à un cours où les données de terrain étaient restituées de manière aussi complète et approfondie. Toutefois, quelques années plus tard, lorsque je fus admise à assister aux séminaires du Laboratoire associé $n^{\circ}$ 221, "Systèmes de pensée en Afrique noire ", j'ai eu l'occasion d'écouter des exposés tout aussi détaillés et passionnés. Je ne citerai ici que le regretté Guy Le Moal (1924-2010), qui a réalisé une documentation écrite et filmique incomparable concernant les Bobo, population de langue mandé du Burkina ${ }^{2}$. Mais il y avait aussi les exposés d'autres personnes, qui sont, heureusement, toujours parmi nous.

En même temps, tout détail ethnographique était, pour Michel Cartry, l'occasion de soulever des interrogations fondamentales. Comme vous le savez, il avait, pendant plusieurs années, étudié la philosophie.

Michel Cartry était exigeant à l'égard du travail écrit de ses étudiants et d'une extrême rigueur quant à la correction du raisonnement. Régulièrement, les weekends et les jours fériés, et singulièrement le 14 juillet, les étudiants inscrits avec lui se présentaient à son domicile. Alors, il se mettait à lire ou à relire à haute voix devant eux le travail qu'ils lui avaient transmis au préalable. Il posait de nombreuses questions et soumettait chaque phrase, chaque enchaînement de phrases, à toutes les critiques possibles. Des étudiants se tenaient dans l'entrée de l'appartement ou bien se promenaient dans le quartier, chacun attendant son tour.

Michel Cartry a ainsi formé, à la méthodologie de terrain et à la pensée anthropologique, de nombreux chercheurs européens et africains. Les Africains étaient, peut-être, proportionnellement plus nombreux à l'époque 
qu'aujourd'hui (problèmes de visas et crises économiques obligent).

Permettez-moi, de nouveau, de citer quelques noms:

Mamadou Lamine Traoré préparait une thèse sur la géomancie au département de philosophie de l'Université Paris IV, Michel Cartry étant membre du jury. Par la suite, Mamadou Lamine Traoré devait être plusieurs fois ministre au Mali - pendant cinq ans, ministre de l'Éducation. En grande partie responsable du développement du rôle des langues nationales dans l'enseignement, il a également fondé un parti politique, Miria (ce mot signifie «pensée » en bambara). Sa thèse, revue, fut publiée à titre posthume fin 2007, sous le titre Philosophie et géomancie ${ }^{3}$.

Moustapha Sanogo et Mamadou Diabaté, tous deux originaires du milieu mandingue de Côte-d'Ivoire, ont soutenu, le premier, un mémoire sur les rites funéraires puis une thèse sur la société initiatique du Koma ${ }^{4}$, le second, un mémoire sur l'initiation de jeunes filles 5 .

Paul Bucumi préparait une thèse sur les épithalames au Burundi, occasion d'exposés dont je n'oublierai jamais le caractère enchanteur'.

Michel Cartry associait à la rigueur méthodologique une grande tolérance intellectuelle et une démarche implicitement pluridisciplinaire.

Personnellement, je lui dois énormément. Alors que d'autres professeurs avaient refusé les thèmes de recherche que je proposais, lui, il m'a non seulement invitée, mais il a insisté pour que je prépare une thèse sous sa direction, sur le sujet qui me tenait à cœur: les castes de l'Afrique occidentale. Par "castes", on entend, dans ce contexte, des groupes endogames d'artisans et musiciens.

C'était la preuve d'une grande ouverture d'esprit, à une époque où parler des castes hors de l'Inde était considéré comme une hérésie, où traiter d'une région aussi vaste (englobant 14 États contemporains) était tenu pour impossible, et où, de plus, l'idée d'étudier l'évolution d'une ins-

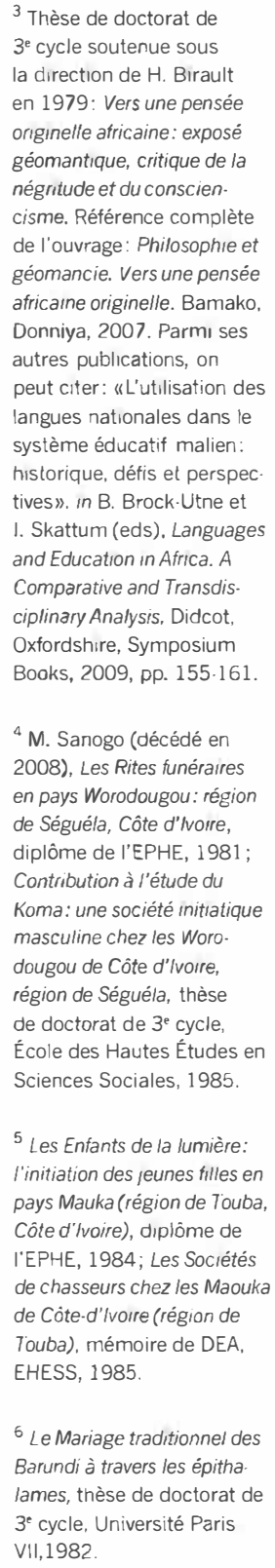

${ }^{3}$ Thèse de doctorat de

$3^{\text {e }}$ cycle soutenue sous la direction de $\mathrm{H}$. Birault en 1979: Vers une pensée origınelle africaine: exposé géomantıque, critique de la négritude et du conscien. cisme. Référence complète de l'ouvrage: Philosophre et géomancie. Vers une pensée africaıne originelle. Bamako, Donniya, 2007. Parmı ses autres publications, on peut citer: "L'utilisation des langues nationales dans le système éducatif malien: historique, défis et perspec. tives». In B. Brock. Utne et I. Skattum (eds). Languages and Education in Africa. A Comparative and Transdis. ciplinary Analysis, Didcot. Oxfordshıre, Symposium Books, 2009, pp. 155.161.

${ }^{4}$ M. Sanogo (décédé en 2008), Les Rites funéraires en pays Worodougou: région de Séguéla, Côte d'lvorre, diplôme de l'EPHE, 1981 ; Contribution à l'étude du Koma: une société initratique masculine chez les Worodougou de Côte d'Ivorre, région de Séguéla, thèse de doctorat de $3^{\text {e }}$ cycle, École des Hautes Études en Sciences Sociales, 1985.

5 Les Enfants de la lumière: l'initiation des jeunes filles en pays Mauka (région de Touba, Côte d'ivoire), diplôme de I'EPHE, 1984; Les Sociétés de chasseurs chez les Maouka de Còte-d'ivorre (région de Touba), mémoire de DEA. EHESS, 1985

${ }^{6}$ Le Mariage traditionnel des Barundi à travers les épitha. lames, thèse de doctorat de $3^{\circ}$ cycle, Université Paris VII, 1982 . 
7 Zeitschrift fur Ethnologie (Berlin) 131(2), 2006; Cahiers d'études africaines (Paris) $46(4), n^{\circ} 184,2006$. titution africaine dans la longue durée était accueillie avec incrédulité.

Lorsque, la rédaction de la thèse achevée, certaines personnes pressenties pour être au jury me recommandèrent d'omettre la comparaison avec l'Inde, Michel Cartry me défendit : "Il faut partir d'un concept ». Pour lui, le comparatisme n'était pas un vain mot. Il pratiquait la comparaison du proche, mais aussi de l'apparemment lointain.

Alors que certains ethnologues au jury m'avaient demandé de retirer mon interprétation des relations à plaisanteries entre clans ou populations comme indices d'alliances politiques passées, Michel Cartry me défendit encore. Récemment, l'interprétation politique de ces relations a fait l'objet de deux colloques, publiés fin 2006 dans deux revues prestigieuses, l'une en France, l'autre en Allemagne?

Michel Cartry m'a également encouragé à poursuivre l'étude de l'autre thème qui m'est cher: les relations entre l'islam et les religions traditionnelles, le lacis de leurs influences réciproques.

Vous aurez compris avec quelles profondes émotion et reconnaissance je rends hommage aujourd'hui à ce maître à penser. 